\title{
Cone-beam CT functional imaging method by using volume integral model
}

\author{
Ying Qian ${ }^{1}$, Boying Zheng ${ }^{2, *}$ \\ ${ }^{1}$ Laboratory of Graphics-Image and Multimedia, Chongqing University of Posts and Telecommunications, China \\ ${ }^{2}$ Laboratory of Graphics-Image and Multimedia, Chongqing University of Posts and Telecommunications, China
}

\begin{abstract}
In order to improve the precision of functional imaging of cone beam computed tomography (CBCT), this paper firstly uses the dynamic contrast enhancement tomography (DCE-CT) of the white rabbit as the measured object and establishes volume integral model to obtain the projection data. Then the optimization method is used to solve the optimal parameter pairs of the voxel time density curve (TDC). Finally, the results of the perfusion are obtained by the deconvolution method. The results show that the TDC correlation coefficient is $83.99 \%$ after, and the maximum of Spearman correlation coefficient of the perfusion parameter is 0.5125 , and the projection time consumption is 7.633 seconds through the volume integral model. It can be seen that the volume integral model is closer to the real projection and it can obtain more accurate perfusion data.
\end{abstract}

\section{INTRODUCTION}

The essence of tumor radiotherapy is to make stronger radioactivity parity radiation, such as $\mathrm{X}$ and $\mathrm{Y}$ ray, focus on one point, and utilize the energy contained in the focus to destroy the tumor cell structure[1].Radiotherapy techniques include anatomical imaging guided therapy and functional imaging guided therapy. Anatomical imaging guidance mainly uses the form of tumor to identify malignant tumor and benign tumor. The image generated by anatomical image guidance technology has good resolution, but it cannot reflect the physiological information of the tissues accurately, which is not conducive to early cancer detection. The functional imaging guidance technology that uses the information of tumor physiology can make up for this deficiency[2]. The principle of CT perfusion imaging is that firstly injecting a proper dose of contrast agent in the vein, then continuously dynamic scanning on the selected level and obtaining the curve of each voxel with time in the region of interest, finally using different mathematical models to find out the functional parameters of the organization[3].

CBCT system is an important equipment for radiation therapy. The ray source and the area detector are synchronized 360 rotation scanning around the measured object. It has the characteristics of small radiation and high imaging precision[4].
In the study of CBCT perfusion imaging, paper [5] proved the possibility of CBCT functional imaging by projection data. Paper [6] used the deconvolution algorithm to establish the physiological model of blood supply, and solved the perfusion parameters in the perfusion imaging. Paper [7-9] designed different projection models to obtain projection data and gained the better perfusion results.

But these projection models were based on the two-dimensional plane, which is not consistent with the actual scanning process of CBCT. Therefore, on the basis of previous research, this paper will use the volume integral model which is closer to the CBCT scanning process to obtain the projection data and complete the CBCT functional imaging.

\section{CBCT SAMPLING PROBLEM AND PERFUSION SIMULATION PROCESS}

The scanning time for CBCT is usually tens of seconds, and it is impossible to obtain the density curve of each voxel at every moment. But according to the kinetic theory, there is no significant change in hemodynamic parameters in 2-3min. Therefore, we can use approximate time density curve to complete perfusion imaging.

\subsection{Obtain the simulated projection data}

\footnotetext{
* Corresponding author: zhengboying@qq.com
} 
In the CT imaging system, the reconstruction algorithm plays a vital role[10].According to the discreteness of voxel and the coverage of ray beam and voxel, the ray coverage model can be divided into point integral model, line integral model, area integral model and volume integral model.

In the volume integral model, the detector is arranged by a square probe. A ray beam is a four pyramid consisting of four vertices of a ray source and a probe. The volume weight of each voxel can be expressed:

$$
W_{i, j}=V_{i, j} / a^{3}
$$

$V_{i, j}$ is the intersecting volume value of $i$ ray beam and $j$ voxel, $a^{3}$ is the volume value of voxel, $V_{i, j}$ is an irregular convex polyhedron, which can be solved by the coordinates of the vertex of the space.

\subsubsection{Simplified projection model}

Taking the center plane of the measured object as the $X O Y$ plane, the radiation source at the rotation angle $\theta=0$ is the origin, and the connection between the ray source and the center of the object is the $\mathrm{Y}$ axis, and the space rectangular coordinate system for the central slice is established(Fig. 1).

Taking the center of the object as the center of rotation and rotating around one circle, we can see that the three-dimensional figure has two characteristics: first, the figure is symmetrical about the plane; second, every 90 degrees per rotation, the intersecting situation is the same. So the model can be simplified, only to consider the part $y \geq 0$ the rotation angle $\theta \in[0,90]$.

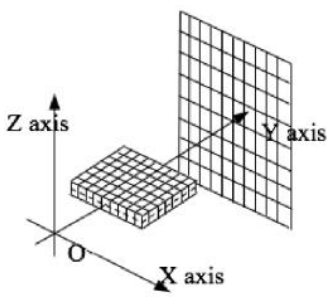

Fig. 1. Space Cartesian coordinate system of central slice.

\subsubsection{Discuss the coverage of slice and ray beam on XOY plane}

The coverage of the ray and voxels on the plane depends on the coverage of two edge rays and voxels. The coverage of single ray and voxel can be divided into full coverage (dark part in Fig. 2), partial coverage (light part in Fig. 2) and no cover (white part in Fig. 2).

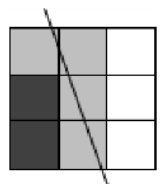

Fig. 2. The coverage of voxel and ray beam on the $X O Y$ plane.

1. Calculate voxel index

The slice is regarded as a $m \times n$ grid, and the ray is regarded as an equation $y=k x+b$, and the two intersection points $p_{1}\left(x_{1}, \mathrm{y}_{1}\right), p_{2}\left(x_{2}, \mathrm{y}_{2}\right)$ of the ray and the slicing border. The coordinate range of the ray can be calculated by two intersection points $X_{\min }=\min \left(X_{1}, x_{2}\right)$, $x_{\max }=\max \left(x_{1}, x_{2}\right), y_{\min }=\min \left(y_{1}, y_{2}\right), y_{\max }=\max \left(y_{1}, y_{2}\right)$. First from the $X$ direction, set $X=X \min +a$, enter the loop condition. when $x<x_{\max },(x, k x+b)$ will be stored in the vertex set \{point\}. (ceil $(x / a)$,floor $(k x+b) / a)$ will be stored in the index set \{index_part\}. Then set $x=x+a$, continue to loop, when $x \geq X_{\max }$ end the loop. Then from Y direction, set $y=y_{\min }+a$, enter the loop condition. When $y<y_{\max },((y-b) / k, y)$ will be stored in the vertex set \{point\}. (ceil $(x / a)$, floor $((k x+b) / a))$ will be stored in the index set \{index_part\}. Then set $y=y+a$, continue to loop, when $y \geq y_{\max }$ end the loop.

Then the voxel index of the full covered is obtained by using the value of the part covered voxel index set and the value of the ray slope $k$. Set $i=1$,enter the cycle condition, when $i \leq m$, all the voxels of $\mathrm{m}$ row are traversed, and the first voxel $(i, j)$ is taken if the line has the voxel passing through the ray. $(i, 1),(i, 2) \ldots(i, j-$ 1 ) will be stored in the index set \{index_full\}. Then set $i=2$, continue the loop, when $i>$ mend the loop. If there is no voxel which is crossed by ray beam in this row, first solved the minimum number of row $i_{\text {min, then set }}$ $i-1$, enter the loop condition. When $i_{\min }<i \leq m$ and $k>0$ or $i \leq i_{\min } \leq m$ and $k \leq 0$, all the voxel index will be stored in the index set \{index_full\},then set $i=2$, continue loop, when $i>m$, end loop.

2. Calculate voxel coordinate set

The coordinates of fully covered voxel are the coordinates of four vertices. And the coordinates of partial covered voxel need to be discussed. Fig. 3 shows a diagram of the relation between a single voxel and a ray line in a partial covered case. $p_{1}, p_{2}, p_{3}, p_{4}$ are the four vertices of the voxel in the $X O Y$ plane.
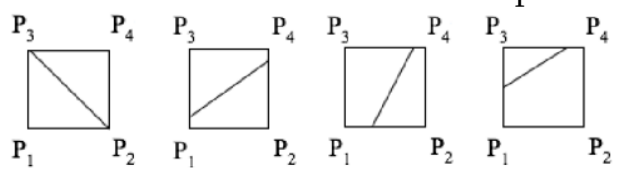

Fig. 3. Graph of relation between partial coverage voxel and ray beam.

Voxel $(m, n)$ has two intersection points $(x, y 1)$, $\left(x_{2}, y_{2}\right)$. Set $\delta x=\left|x_{1}-x_{2}\right|, \delta y=\left|y_{1}-y_{2}\right|$. When $\delta x=a$ and $\delta y=a$, the ray line coincides the diagonal line (as in Fig. 3 (a)). If $k>a$, record $p 3$,else $p$; When $\delta x=a$ and $\delta y=a$, the ray 
line cross over the voxel from $\mathrm{X}$ direction (as in Fig. 3 (b)). If $k>0$, record $p_{3}$ and $p_{4}$,else $p_{1}$ and $p_{2}$; When $\delta x \neq a$ and $\delta y=a$, the ray line cross over the voxel from $Y$ direction (as in Fig. 3 (c)). If $k>0$, record $p$ and $p_{3}$, else $p 2$ and $p_{4}$; When $\delta x \neq a$ and $\delta y \neq a$, the ray line cross over the adjacent edges of the voxel (as in Fig. 3 (d)), it needs to discuss voxel $(m-1, n)$. When $(m-1, n)$ is crossed over by ray line, if $k>0$, record $p 3$,else $p 1$; When $(m-1, n)$ does not exist or is not crossed over by ray line, if $k>0$,record $p_{1}, p_{3}, p_{4}$ else $p_{2}, p_{3}, p_{4}$.

3 . Calculate coverage of ray beam and voxel

Calculating the coverage of the ray beam and the voxel by the set operation. If a vertex set of $h_{1}$ is $A$, and a vertex set of $h$ is $B$, the result is $C=(A+B)-(A-B)$.

\subsubsection{Discuss the coverage of slice and ray beam in three-dimensional space}

In three-dimensional space, the coverage can be divided into three types: no cutting, partial cutting and complete cutting.

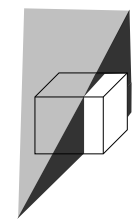

(a)

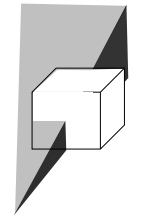

(b)

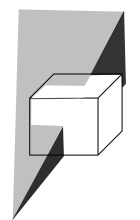

(c)
Fig. 4. Coverage of ray beams and voxels in space.

When no cutting (as shown in Fig. 4 (a)), the height of the upper slope of the beam is higher than the upper surface of the voxel, so the coordinates on the upper surface of the beam are all on the $z=a / 2$ plane; When complete cutting (as shown in Fig. 4 (b)), the height of the upper slope of the beam is lower than the upper surface of the voxel, so the coordinates on the upper surface of the voxel are all on the plane where the slope is located; When partial cutting (as shown in Fig. 4 (c)), the intersection of the upper slope of the beam and upper surface of the voxel is a cutting line. So partial cutting can be split into no cutting and complete cutting.

In the case of complete cutting, the $z$ value in the surface coordinates of the voxel can be solved by the principle of similar triangle. Set point $M^{\prime}\left(x_{M}, y_{M}, 0\right)$ which is on the $X O Y$ plane is the projection point $M(X M, Y M, Z M)$ which is on the plane of the slope. The length of probe is $d$, the central point of detector is $D\left(x D, y_{D}, 0\right)$, source is $S(x s, y s, 0)$, distance between source and detector is 1 . Linking $N$ and $M$ :

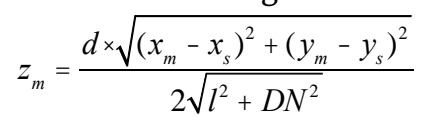

In the case of partial cutting, the expression of the cutting line and the voxel index of the cutting line are first required. Set projection line of cutting line is $g=f(x)$, it is perpendicular to angular bisector of rays beam on the XOY plane and crossing $\operatorname{point}\left(\left(x_{d}+X_{s}\right) \cdot a / d,\left(y_{d}+y_{s}\right) \cdot a / d\right)$, so the $g=f(x)$ is:

$$
g=\frac{x_{D} x_{S}}{y_{D} y_{S}}\left[\frac{\left(x_{D}+x_{S}\right) \times a}{d} x\right]+\frac{\left(y_{D}+y_{S}\right) \times a}{d}
$$

Then using the method of calculating the partial coverage index and coordinate (as 2.1.2) to obtain the $z$ value. If $z<a / 2$, the real $z$ value is $(x, y, z)$,else $(x, y, a / 2)$.

\subsubsection{Calculate the intersecting volume of voxel and ray beams}

The part of the ray beam and the voxel is an irregular convex polyhedron. The volume of a convex polyhedron in a three-dimensional space is equal to the sum of the sum of each polygon on which each of its surfaces takes a positive determinant. [14] ,that is, for convex polyhedron $A_{0} A_{1} A_{2} \ldots A_{N}$, the volume is:

$$
V\left(A_{0} A_{1} A_{2} \cdots A_{N}\right)=\sum d\left(A_{0} A_{1} A_{2} \cdots A_{N}\right) / 6
$$

\subsubsection{Optimize volume integral model using GPU parallel computing}

The traditional CPU executes the program in a serial way, which is mainly used to control logic. And GPU uses multithreading to calculate large and dense data, and its speed and throughput are higher than CPU.

Since the computation of each ray beam's projection data on detector is independent of each other in volume integral model, we can use GPU parallel computing to optimize volume integral model and reduce projection time.

\subsection{Obtain the approximate time density curve}

Paper [13] confirmed that the time density curve obtained by the contrast agent regiment in functional CT is usually described by a gamma function. In Paper [5], the reverse tangent function is introduced to simulate the trend of the TDC curve reaching its peak. Paper [7] considers the initial density value before the contrast agent is not injected to characterize the actual hemodynamic condition of the organism. The model expression is:

$$
X(t)=\left\{\begin{array}{cr}
P & ,(t<T) \\
(t-T)^{a} \times e^{-b(t-T)}+c \times \arctan (t-T)+P,(t \geq \mathrm{T})
\end{array}\right.
$$

$X(t)$ is the density value of voxel changing with time; $P$ indicates the density of objects when no contrast agent is injected; $t$ is the time after injection of contrast agent; $T$ is the time when the contrast 
agent enters the scanning layer; $a, b$ and $c$ are the model parameters.

\subsection{Obtain CBCT perfusion parameters}

The contrast agent in artery indicates $\mathrm{Ca}(t)$. After entering the tissue, the concentration of contrast agent will rise sharply in a short time, then smooth, and finally return to the state of no perfusion.

The concentration changes of the contrast agent in the tissue $Q(t)$ can be calculated by the product of the tissue blood flow BF and the tissue residency function $R(t)$,and the TDC of the tissue can be detected by the CT device.

$$
Q(t)=B F \times C a(t) \quad R(t)
$$

The relation between $R(t)$ and time $t$ is:

$$
R(t)=\left\{\begin{array}{l}
1,0 \leq t \leq M T T \\
E^{2} e^{\frac{B F \cdot E}{V_{e}}(2 t 3 M T T)}, t \geq M T T
\end{array}\right.
$$

MTT indicates the average time for contrast agents to flow out of the veins after the artery enters the tissue. $V_{e}$ is volume of intercellular space. $E$ is associated with surface permeability PS and blood flow BF:

$$
E=1 \quad e^{P S / B F}
$$

Finally, blood volume BV can be obtained by using the blood flow BF and the average of time multiplied by time MTT:

$$
B V=B F \times M T T
$$

\section{EXPERIMENT AND ANALYSIS}

\subsection{Experimental materials and preparation}

The experimental image is a New Zealand white rabbit with VX2 tumor implanted in legs at the princess Margaret Hospital of Toronto. After two weeks of tumor implantation, 4 groups of 120 DICOM format sequence images (Fig. 5) were obtained, and the specific parameters were as Table 1 .

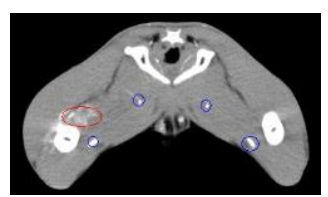

Fig. 5. DCE-CT sequence map of VX2 type tumor.

Table 1. Experimental material parameters.

\begin{tabular}{|c|c|}
\hline Name & value \\
\hline Scanning speed (s/circle) & 1 \\
\hline
\end{tabular}

\begin{tabular}{|c|c|}
\hline $\begin{array}{c}\text { The distance from the ray source } \\
\text { to the axis of rotation(mm) }\end{array}$ & 541 \\
\hline $\begin{array}{c}\text { The distance between the ray } \\
\text { source and the detector }(\mathrm{mm})\end{array}$ & 949 \\
\hline Image pixel size & $512 * 512$ \\
\hline
\end{tabular}

As a result of the calculation of speed, a tumor area (9 99 pixel size), such as Fig. 6, is selected as the object of study.

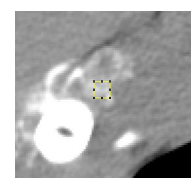

Fig. 6. Selected regions of interest in the tumor.

The experimental simulation environment parameters are shown in Table 2.

Table 2. Simulation environment parameters

\begin{tabular}{|c|c|}
\hline Name & value \\
\hline Platform & Windows 10 \\
\hline Processor & Intel(R) Core(TM) i5-4210H \\
\hline Software tool & Matlab R2017a \\
\hline Graphics cards & NVDIA GeForce GTX 960M \\
\hline
\end{tabular}

\subsection{Projection data and cost}

The contrast point integral model [7], the line integral model [8] and the area integral model [9] are used to get the CBCT projection sine of the second slices, as shown in Fig. 7.

The figure under the visible volume integral model reduces most of the saw tooth artifact, and the image quality is better than the other three models.

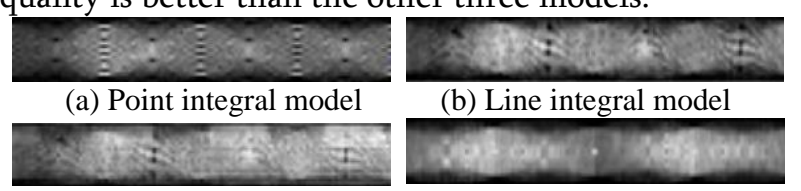

(c) Area integral model (d) Volume integral model

Fig. 7. The sine map of CBCT projection data.

Table 3 shows the cost of projection under the four models. The volume integral model brings a lot of computational cost while improving the accuracy of the image. However, after the use of GPU optimization, the calculation of the volume integral model is greatly reduced.

Table 3. Projection costs of various projection models (units: seconds) 


\begin{tabular}{|c|c|c|c|c|}
\hline $\begin{array}{c}\text { Projection } \\
\text { model }\end{array}$ & Slice 1 & Slice 2 & Slice 3 & Slice 4 \\
\hline Point integral & 0.1061 & 0.1635 & 0.1220 & 0.1535 \\
\hline Line integral & 1.2657 & 1.0982 & 1.2017 & 1.5211 \\
\hline Area integral & 3.1228 & 2.8452 & $\begin{array}{c}2.8412 \\
1\end{array}$ & 3.4290 \\
\hline $\begin{array}{c}\text { Volume } \\
\text { integral } \\
\text { (CPU) }\end{array}$ & 9.0612 & 8.9413 & 8.8875 & 9.2189 \\
\hline $\begin{array}{c}\text { Volume } \\
\text { integral } \\
\text { (GPU) }\end{array}$ & 7.5361 & 7.8245 & 7.3608 & 7.8105 \\
\hline
\end{tabular}

\subsection{Correlation coefficient of TDC}

The optimization method is used to solve the simulated TDC. The reasonable parameter range are $a \in[2.50,3.00], b \in[0.180,0.210], c \in[15,35], T \in[10,20]$. In order to quantify the similarity between the simulated TDC and the real TDC, the correlation coefficient is used to evaluate it.

$$
r=\frac{\operatorname{Cov}(X, Y)}{\sqrt{D(X)} \times \sqrt{D(Y)}}=\frac{E(X Y) E(X) E(Y)}{\sqrt{E\left(X^{2}\right) E^{2}(X)} \times \sqrt{E\left(Y^{2}\right) \quad E^{2}(Y)}}
$$

$r$ represents correlation coefficient, the range is between -1 and 1, $X$ represents TDC of a voxel, $Y$ represents the real TDC of a voxel, the larger the absolute value of $r$ is, the greater the similarity of two variables.

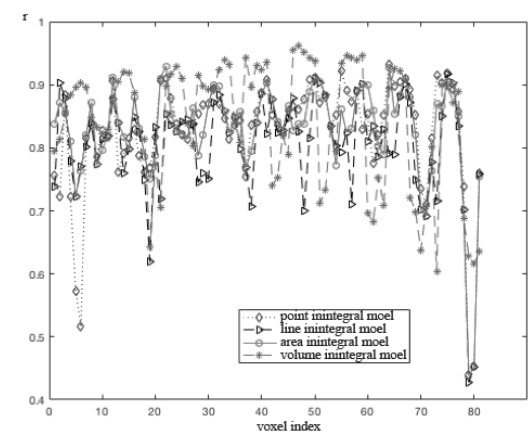

Fig. 8. correlation coefficient of real TDC and simulated TDC under each projection model.

Taking second slices as an example, the correlation coefficient under the four models is shown in Fig. 8. The average correlation coefficient of point, line, surface and volume model is 0.804024983 , $0.825983271,0.829104662,0.839869259$, and the visible volume integral model is better than the other three models.

\subsection{Correlation coefficient of perfusion parameters}

The correlation between the simulated perfusion results and the actual perfusion results was evaluated using the Spearman correlation coefficient. The Spearman correlation coefficient does not require the distribution of the original data, and the expression is:

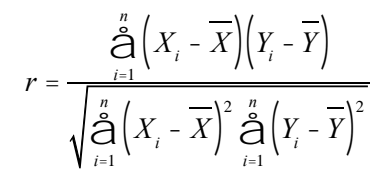

$r$ represents the correlation coefficient ranging from -1 to 1 . $X$ represents the simulated perfusion parameter value, and $Y$ represents the true perfusion parameters. The greater the correlation coefficient $r$, the greater the correlation.

Table 4. The mean of Spearman correlation coefficient

\begin{tabular}{|c|c|c|c|c|}
\hline $\begin{array}{c}\text { Projection } \\
\text { model }\end{array}$ & MTT & PS & BV & BF \\
\hline Point integral & 0.3475 & 0.1677 & 0.4388 & 0.0946 \\
\hline Line integral & 0.4281 & 0.1464 & 0.4936 & 0.2172 \\
\hline Area integral & 0.4477 & 0.2222 & 0.4747 & 0.2238 \\
\hline $\begin{array}{c}\text { Volume } \\
\text { integral (CPU) }\end{array}$ & 0.4955 & 0.2540 & 0.5125 & 0.2976 \\
\hline $\begin{array}{c}\text { Volume } \\
\text { integral (GPU) }\end{array}$ & 0.3475 & 0.1677 & 0.4388 & 0.0946 \\
\hline
\end{tabular}

\section{Conclusion}

This paper proposes the method of cone beam CT imaging using volume integral model. First using the DCE-CT image as the measured object and using point integral model, line integral model, area integral model and volume integral model to complete the projection process. Then solving the TDC curve by a voxel time density model. Finally obtaining the perfusion parameters by the hemodynamic model.

The experimental results show that the accuracy of the perfusion parameters is the highest under the volume integral model, and the corresponding projection consumption is also reduced after using the GPU acceleration. It proves the advantage of the volume integral model in CBCT functional imaging.

\section{References}

1. G.L. Jiang, Modern radiotherapy for tumor (Shanghai Science and Technology Press, Shanghai, 2009)

2. J.L. Hu, JournalTCM, 03, 305-306 (2011) 
3. Z.Z. Hu, Y.F. Huang, J.L. Zeng, et al, Journal of clinical and Experimental Medicine 10, 1153-1154 (2011)

4. W.C. Scarfe, A. G. Farman, P. Sukovic. J CAN DENT ASSOC 72, 75(2006)

5. Y. Qian, Q. Zhou, Q.J. Wei, ACTA ACADEMIAE MEDICINAE MILITARIS TERTIAE 32, 17321735 (2010)

6. S.Y. Wang, Y Qian. Journal of Clinical Rehabilitative Tissue Engineering Research 14, 5573-5576 (2010)

7. Y. Qian, W.F. Yang, JCA 31, 1242-1244 (2011)

8. Y. Qian, J.Q. Qin, JCA s2, 263-266 (2015)

9. Y. Qian, T.T. Liao, JCA 36, 3429-3435 (2016)

10. J.L. Chen, B. Yan, L. Li, et al, CT Theory and Applications 23, 317-328 (2014)

11. S. Ha, A. Kumar, K. Mueller. The 13th International Meeting on Fully Three-Dimensional Image Reconstruction in Radiology and Nuclear Medicine (2015)

12. D. B. Kirk, W. M. Hwu, Programming Massively Parallel Processors: A Hands-on Approach. (China Machine Press, Beijing, 2013)

13. K. A. Miles, EUR J CANCER 38, 79-84 (2002)

14. J. Wei 1997. Journal of Huzhou Teachers College (Natural Science) 5, 67-73 (1997) 